Structural Eng. / Earthquake Eng. Vol.5. No.2, 397s-400s. October 1988/Technical Note

Japan Society of Civil Engineers (Proc. of JSCE No.398/I-10)

\title{
EFFECT OF TURBULENCE ON THE MODAL INTERACTION IN GALLOPING OF STRUCTURE WITH TWO CLOSELY-SPACED NATURAL FREQUENCIES
}

\author{
By Phoonsak PHEINSUSOM* and Yozo FUJINO**
}

\begin{abstract}
Effect of turbulent wind on modal interaction in galloping of structure with two closely-spaced natural frequencies is studied. Numerical simulation indicates that the presence of certain intensity of turbulence causes the modal interaction, i. e. the dominant vibration mode alternately changes from one mode to the other in time domain. The modal responses seemingly are the combination of galloping response with time-varying amplitude and the random buffeting responses. This is also confirmed by wind tunnel observations.

Keywords: buffeting, closely-spaced natural frequencies, galloping, modal interaction, numerical simulation, turbulent wind, wind tunnel testing
\end{abstract}

\section{INTRODUCTION}

Across-wind galloping of the structure with two closely-spaced natural frequencies in uniform wind flow has been studied by the authors ${ }^{1}$. It was found analytically as well as experimentally that when galloping oscillation occurs in the structure with symmetrical properties, its steady-state motion consists of a single mode galloping, and the modal amplitude depends on its structural damping. The initial conditions or the external disturbances determines the mode which will actually attain steady state.

Actual wind is turbulent in nature and the structure is, therefore, subjected not only to the self-excited wind force but also to the fluctuating buffeting wind force. It is of interest whether the presence of buffeting force can produce sustained interaction of the two vibration modes, which may be classified here as modal interaction.

Effect of presence of the buffeting wind force on the galloping of structure with closely-spaced natural frequencies is studied analytically as well as experimentally.

\section{ANALYTICAL METHOD}

We consider the original proposal of the Higashi-Kobe cable-stayed bridge tower (Fig. 1) subjected to the wind force. The structure is assumed to be a linear elastic proportionally-damped system. The external wind force, $F_{Y}$ is assumed to consist of two components : self-excited galloping force, $F_{g}$, and stationary fluctuating buffeting wind force in direction perpendicular to wind flow, $F_{b}$ :

$F_{g}=0.5 \rho U^{2} D C_{L d}$

$F_{b}=0.5 \rho U\left\{2 B u C_{L}+B v\left(d C_{L} / d \alpha\right)_{\alpha=0}+D v C_{D}\right\}$

* Member of JSCE, M. Eng., Graduate student, Dept. of Civil Eng., Univ. of Tokyo (Bunkyo Tokyo 113)

** Member of JSCE, Ph. D., Assoc. Prof., Eng. Res. Int., Univ. of Tokyo (Bunkyo Tokyo 113) 
in which $\rho=$ air density, $U=$ mean wind velocity. $B, D=$ dimension of structure in along and across wind directions, respectively. $u, v=$ turbulent velocity fluctuation in along-wind and across-wind directions, respectively. $C_{D}, C_{L}=\mathrm{drag}$ and lift coefficients in turbulence wind at $\alpha$ $=0$, respectively, and $d C_{L} / d \alpha=$ derivative of lift coefficient in turbulence wind with respect to angle of wind attack, $\alpha$.

Under quasi-steady assumption, $C_{L d}$ are written in polynomials function of structural velocity, $\dot{Y}$ as

$$
C_{L d}=\left\{A_{1}\left(\frac{\dot{Y}}{U}\right)+A_{2}\left(\frac{\dot{Y}}{U}\right)^{2}+A_{3}\left(\frac{\dot{Y}}{U}\right)^{3}\right\}
$$

where $A_{i}=$ aerodynamic force coefficients.

Using the same procedures used in Ref. 1), modal equations of motion for structure under both galloping and buffeting forces are obtained as

$$
\begin{aligned}
& \ddot{y}_{1}+\omega_{1}^{2} y_{1}=\alpha_{1} \dot{y}_{1}+\alpha_{2} \dot{y}_{1}^{2}+\alpha_{3} \dot{y}_{1} \dot{y}_{2}+\alpha_{4} \dot{y}_{2}^{2}+\alpha_{5} \dot{y}_{1}^{3}+\alpha_{6} \dot{y}_{1}^{2} \dot{y}_{2}+\alpha_{7} \dot{y}_{1} \dot{y}_{2}^{2}
\end{aligned}
$$

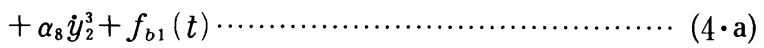

$$
\begin{aligned}
& \ddot{y}_{2}+\omega_{2}^{2} y_{2}=\beta_{1} \dot{y}_{2}+\beta_{2} \dot{y}_{1}^{2}+\beta_{3} \dot{y}_{1} \dot{y}_{2}+\beta_{4} \dot{y}_{2}^{2}+\beta_{5} \dot{y}_{1}^{3}+\beta_{6} \dot{y}_{1}^{2} \dot{y}_{2}+\beta_{7} \dot{y}_{1} \dot{y}_{2}^{2}
\end{aligned}
$$

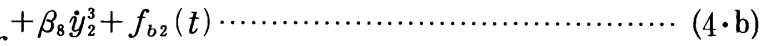

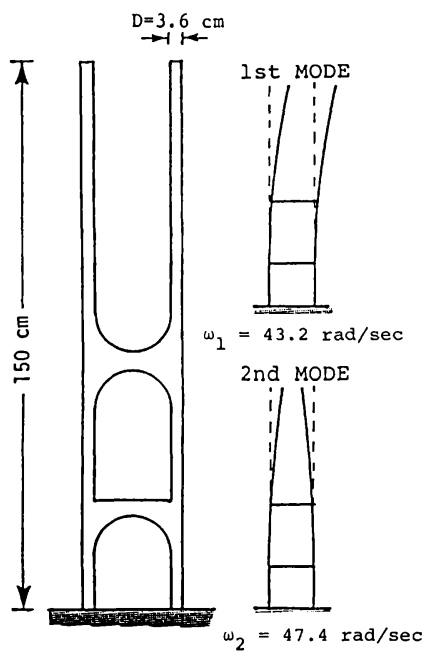

Fig. 1 Higashi-Kobe cable-stayed bridge tower's model (see Ref. 1) for modal and model properties).

where $f_{b i}=\int F_{b} \gamma_{i} d x=i$-th modal random wind force, $\gamma_{i}=i$-th modal shape. $y_{i}=i$-th modal amplitude, $\omega_{i}$ $=i$-th modal circular natural frequencies. The parameters $\alpha_{1} \sim \alpha_{8}$ and $\beta_{1} \sim \beta_{8}$ are defined in Eq. $(7 \cdot \mathrm{a}) \sim$ $(7 \cdot s)$ (Ref. 1)) and they are functions of mode shapes, cross-section, wind velocity and aerodynamic force coefficients $\left(\gamma_{1}, \gamma_{2}, D, U\right.$ and $A_{i}$, respectively). Since, the exact values of aerodynamic force coefficients in turbulent flow $\left(A_{1}, A_{2}\right.$ and $\left.A_{3}\right)$ of the model in Fig. 1 are not measured in wind tunnel, then the aerodynamic force coefficients of $2: 3$ rectangular cross-section in turbulent wind obtained in Ref. 2) are used, i. e. $A_{1}=1.35, A_{2}=0.0$ and $A_{3}=-40.02$. Note that Eqs. $(4 \cdot a)$ and $(4 \cdot b)$ are basically identical to Eqs. $(6 \cdot a)$ and $(6 \cdot b)$ in Ref. 1$)$, except the modal buffeting force terms of $f_{b 1}(t), f_{b 2}(t)$.

Since analytical solution for Eqs. $(4 \cdot a)$ and $(4 \cdot b)$ is very difficult to obtain, the computer simulation is conducted. Buffeting forces, $F_{b}$ is computed by simulating of fluctuating wind components, $u$ and $v$. The power spectrum density functions of grid-generated turbulence in Ref. 3) are used. Note that the modal buffeting forces $f_{b 1}(t)$ and $f_{b 2}(t)$ are statistically independent because of the modal shape properties of $\gamma_{1}$ and $\gamma_{2}$, which are symmetric and anti-symmetric, respectively as shown in Fig. 1. Time history responses of $y_{1}(t)$ and $y_{2}(t)$ are calculated by the Newmark integration method.

\section{SIMULATION RESULTS}

Time history responses $y_{1}$ and $y_{2}$ of $1: 100$ scaled model of the tower (Fig. 1) are computed for various intensities of the buffeting force, $F_{b}$. Some examples of the results for the mean wind velocity $U>$ $U_{c r 1}, U_{c r 2}$ are presented in Fig. 2. Note that $U_{c r i}$ is the onset wind velocity of the $i$-th mode galloping.

It was found that under rather low intensity of buffeting forces $\left(I_{u}=5 \%\right)$, the structure vibrates mainly in the first (or second) mode with the small random amplitude of the second (or first) mode and there is no switch in dominant vibration mode, i. e. no modal interaction. An example of the dominant first mode motion is given in Fig. 2 (a).

Under large intensity $\left(I_{u}=11 \%\right)$, the two modes interact in such a way that they exhibit peaks of comparable magnitude alternately in time domain as shown in Fig. 2(b). Simultaneous large amplitudes of the first and the second modes are hardly observed. This modal interaction phenomenon can be more clearly observed by means of modal energy, $E_{i}=0.5\left(\dot{y}_{i}^{2}+w_{i}^{2} y_{i}^{2}\right), i=1,2$. The ratio between modal energy $\left(E_{1}\right.$ or $\left.E_{2}\right)$ by total energy $\left(E_{T}=E_{1}+E_{2}\right)$ of the responses in Fig. 2(b) are presented in Fig. 3(a). Modal energy ratios, $E_{1} / E_{T}$ and $E_{2} / E_{T}$ for the two independent random responses simulated by disregarding the 


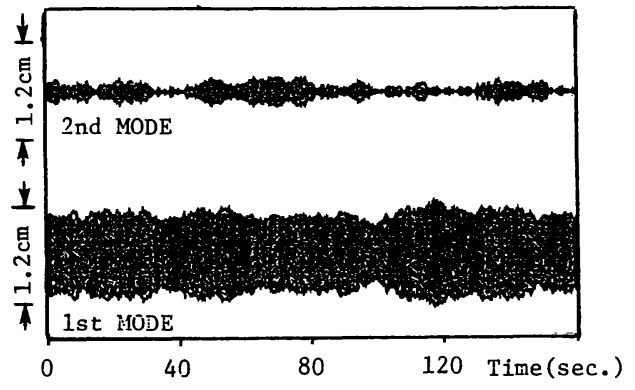

(a) Modal responses at $\mathrm{Iu}=5 \%$

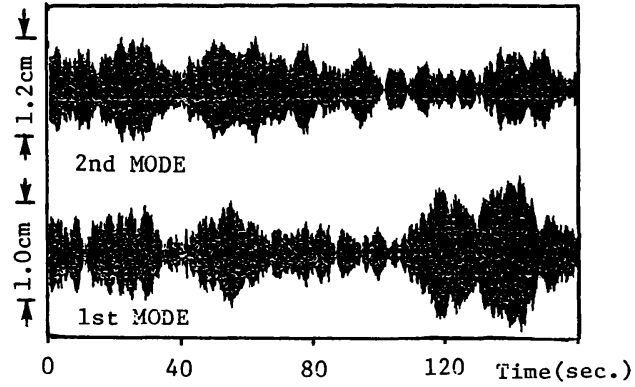

(b) Modal responses at $\mathrm{Iu}=11 \%$

Fig. 2 Simulated modal responses at $U>U_{c r 1}, U_{c r 2}:$ (a) at low buffeting intensity, $I_{u}=5 \%$, (b) at high buffeting intensity, $I_{u}=11 \%$.

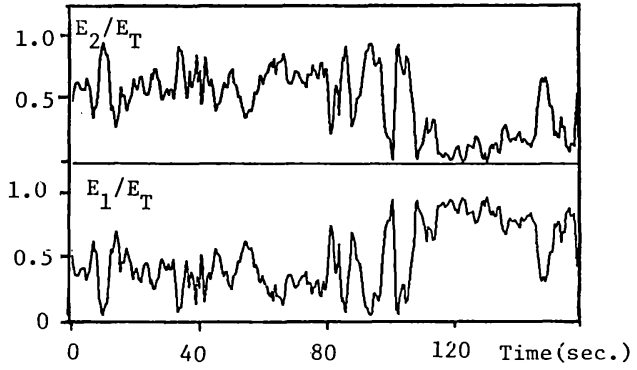

(a) Modal energy ratios of responses in Fig.2(b).

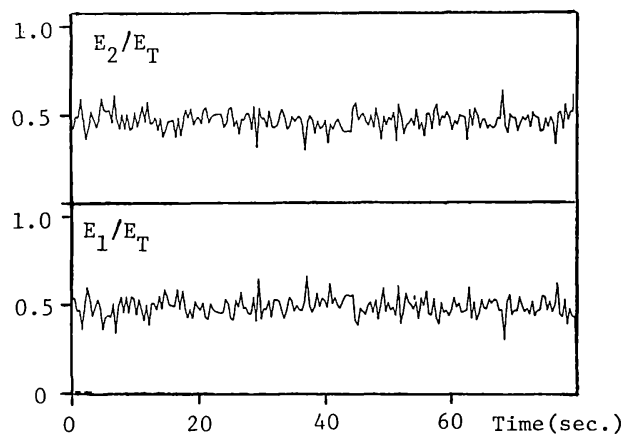

(b) Modal energy ratios of simulated independent fluctuated responses.

Fig. 3 Modal energy ratios of the simulated responses.

non-linear coupled terms in Eq. (4) are presented, for reference, in Fig. 3(b). As shown in Fig. 3(b), even the peak of each modal energy ratio exists alternately ; however, change of model energy of responses shown in Fig. 3(a) is more profound than that shown in Fig. 3(b). The alternate change of the peak in Fig. 3 (a) indicates the alternate change of the dominant vibration mode, i. e. modal interaction. This modal interaction may be caused by the nonlinear coupling terms between the first and the second modes in modal equations of motion.

The Probability Density Functions (PDFs) of the responses shown in Fig. 2(b) are presented in Fig. 4. Both of the PDFs are neither the normal distribution (indicating buffeting responses) nor the arcsine $\left(\sin ^{-1}\right)$ distribution (indicating steady-state sinusoidal response). The PDFs are instead the combination of normal and arcsine distributions, indicating that buffeting and galloping responses are superimposed.

\section{COMPARISON WITH WIND TUNNEL EXPERIMENTS}

The wind tunnel experiment using the $1: 100$ scaled tower model (Fig. 1) under turbulent flow was also performed. Turbulent flow was generated by the $\operatorname{grid}^{3)}$. Examples of modal responses are shown in Fig. 5.

Modal responses at high wind velocity are presented in Fig.5(a). Galloping with steady

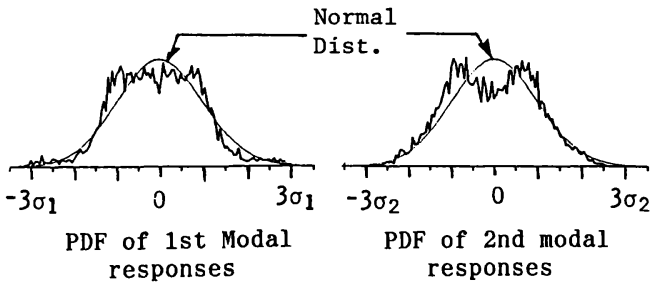

Fig. 4 PDFs of simulated responses in Fig. 2(b) $\left(\sigma_{1}=0.63 \mathrm{~cm}, \sigma_{2}=0.48 \mathrm{~cm}\right)$. 


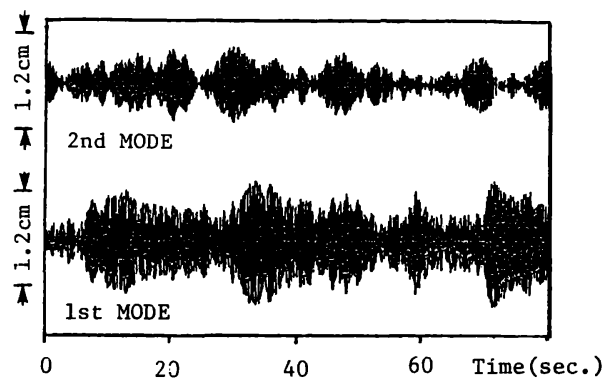

(a) Modal responses at $U=5.9 \mathrm{~m} / \mathrm{s}$

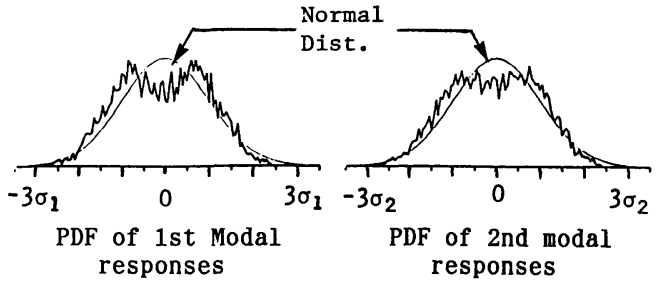

(b) PDFs of modal responses at $U=5.9 \mathrm{~m} / \mathrm{s}$

Fig. 5 Modal responses and their PDFs obtained from wind tunnel test at high wind velocity $\left(U=5.9 \mathrm{~m} / \mathrm{s}, \sigma_{1}=0.32 \mathrm{~cm}, \sigma_{2}=0.18 \mathrm{~cm}\right)$.

amplitudes is not clearly observed ; however, the PDFs (Fig. 5(b)) of each modal response is some what different from the normal distribution and appears instead as a combination of the normal and arcsine distributions, indicating the existence of buffeting as well as galloping. This is also observed from the modal energy, as shown in Fig. 6, that they exhibit peaks almost alternately in time domain and that there is modal interaction at high wind velocity. The overall characteristics are

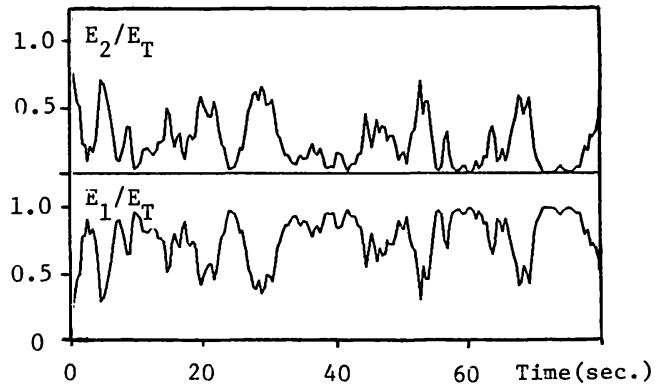

Fig. 6 Modal energy ratio of modal responses in Fig. 5(a). similar to those obtained by simulation. At lower wind velocity, the PDFs of responses were almost similar to the normal distributions and modal responses almost consisted of the buffeting responses.

\section{SUMMARY}

Modal interaction of galloping in a linear elastic proportionally-damped system with two closely-spaced natural frequencies under turbulent wind has been analytically and experimentally studied. In the presence of certain amount of buffeting force as well as galloping force, the modal interaction occurs between the closely-spaced two modes and the dominant vibration mode changes from one mode to the other almost alternately in time domain. The results in wind tunnel experiments using the three-dimensional tower model confirm these simulation results.

\section{ACKNOWLEDGEMENT}

The authors would like to thank Prof. Manabu ITO and Dr. Benito PACHECO, Univ. of Tokyo for their comments and suggestions. This study was partially supported by the Grant-in-Aids for Scientific Research, No. 61460156 .

\section{REFERENCES}

1) Pheinsusom, P. and Fujino, Y. : Galloping of Structure With Two Closely-spaced Natural Frequencies, Proc. of JSCE, No. 392/ I -9, pp. 215 225, Apr. 1988.

2) Novak, M. and Tanaka, H. : Effect of Turbulence on Galloping Instability, Jour. of the Engineering Mechanical Division, ASCE, EM 1, pp. 27 47, Feb. 1974.

3) Nakajima, T. : Aeroelastic Behaviours of Rectangular Bluff Body in Turbulent Wind Generated By Grid, Master Thesis, University of Tokyo, 1974. 